\title{
A linear algorithm for Brick Wang tiling
}

\author{
Alexandre Derouet-Jourdan ${ }^{\mathrm{a}}$, Shizuo Kaji ${ }^{\mathrm{b}, *}$, Yoshihiro Mizoguchi $^{\mathrm{c}}$ \\ ${ }^{a}$ OLM Digital Inc. \\ ${ }^{b}$ Yamaguchi University / JST PRESTO \\ ${ }^{c}$ Kyushu University
}

\begin{abstract}
The Wang tiling is a classical problem in combinatorics. A major theoretical question is to find a (small) set of tiles which tiles the plane only aperiodically. In this case, resulting tilings are rather restrictive. On the other hand, Wang tiles are used as a tool to generate textures and patterns in computer graphics. In these applications, a set of tiles is normally chosen so that it tiles the plane or its sub-regions easily in many different ways. With computer graphics applications in mind, we introduce a class of such tileset, which we call sequentially permissive tilesets, and consider tiling problems with constrained boundary. We apply our methodology to a special set of Wang tiles, called Brick Wang tiles, introduced by Derouet-Jourdan et al. in 2015 to model wall patterns. We generalise their result by providing a linear algorithm to decide and solve the tiling problem for arbitrary planar regions with holes.
\end{abstract}

Keywords: Wang tile, graphical model, satisfiability problem

2010 MSC: 05B45, 52C20, 68R10

\section{Introduction}

Wang tiles are a class of formal systems introduced by Hao Wang in 1961 [15]. A Wang prototile can be thought of as a square tile with a colour on each side. Once a set of such prototiles is given, copies of prototiles are placed side by side so that the colours of common edges match. Given a finite set of Wang prototiles, the domino problem asks whether a whole plane can be tiled with copies of them, and was proved undecidable by R. Berger in 1966 [2]. There are also sets of Wang prototiles which can tile the plane but only aperiodically $([2,9,4,4,8])$.

In practical applications, bounded planar regions are usually considered, with which the corresponding domino problem is obviously decidable. Moreover, sets of Wang prototiles are normally restricted to a permissive class so that there exist many solutions in general. In this case, it is more reasonable to ask for efficient algorithms to decide tilability with constrained boundary and give solutions if they exist. We make clear our framework in \$2. In general, the problem is known to be NP-complete [12], so we restrict ourselves to a certain sub-problem. Namely, we deal with a specific setting which arises in computer graphics applications such as procedural synthesis of textures, height fields, and sampling points [3, 10, 14]. In this case, sets of Wang prototiles are often sequentially permissive (Def. 1), and we have a general strategy described in Lemma 9. In \$3, we specialise to the tiling problems with the Brick Wang tiles (Def.

\footnotetext{
${ }^{*}$ Corresponding author

Email addresses: alexandre.derouet-jourdan@olm.co.jp (Alexandre Derouet-Jourdan), skaji@yamaguchi-u.ac.jp (Shizuo Kaji), ym@imi . kyushu-u.ac.jp (Yoshihiro Mizoguchi)
} 
10) introduced in [6] to generate wall pattern textures. It is proved in [6] that any rectangle region greater than the $2 \times 2$ square with any boundary colour condition can be tiled. This result was then formally verified and implemented in [13] using the Coq proof assistant [1]. We generalise the result by giving a complete characterisation of the set of regions which can be tiled with any given boundary constraints. In \$4, we provide a linear time algorithm for the Brick Wang tiling problem, with an implementation with source codes at [5].

\section{Problem setting}

We begin with introducing a type of Wang tiling problem which can be regarded as an extension problem or a tiling problem with boundary constraints. Then, we consider a class of Wang prototiles having a special property (1) and discuss a strategy for a tiling algorithm (Lemma 9).

We first define the object to be tiled as an enriched graph.

Definition 1. A board is a finite undirected graph $G=\left(V \cup\left\{v_{0}\right\}, E\right)$ with a distinguished vertex $v_{0}$ called the constrainer which satisfies the following:

- at each $v \in V$, the set of edges $N(v)$ adjacent to $v$ is ordered: $N(v)=\left\{e_{1}(v), \ldots, e_{\operatorname{deg}(v)}(v)\right\}$, where $\operatorname{deg}(v)$ is the degree of $v$

- the full sub-graph on $V$ is connected.

Vertices in $V$ are called cells. We often identify a board with its underlying graph when there is no confusion.

Figure 1 shows an intuitive correspondence between our graphical formalisation and the usual Wang tile.
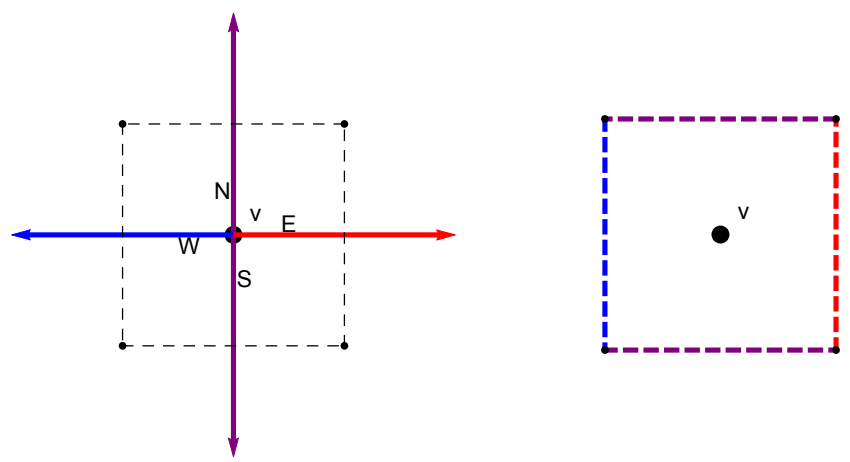

Figure 1: Graph vs Wang tile

Definition 2. A tiling problem $(G, C, W)$ consists of

- a board $G=\left(V \cup\left\{v_{0}\right\}, E\right)$

- a set of colours $C$

- a set of prototiles $W=\bigcup_{k=1}^{\infty} W_{k}$, where $W_{k} \subset C^{k}$.

A tiling of a subset $F \subset E$ is a map $\tau: F \rightarrow C$ satisfying $\left(\tau\left(e_{1}(v)\right), \ldots, \tau\left(e_{\operatorname{deg}(v)}(v)\right)\right) \in W$ for any $v \in V$ with $N(v) \subset F$. It is said to be full if $F=E$. A tiling $\tau^{\prime}: F^{\prime} \rightarrow C$ is called an extension of another tiling $\tau: F \rightarrow C$ with $F \subset F^{\prime}$ if $\tau(e)=\tau^{\prime}(e)$ for any $e \in F$. 


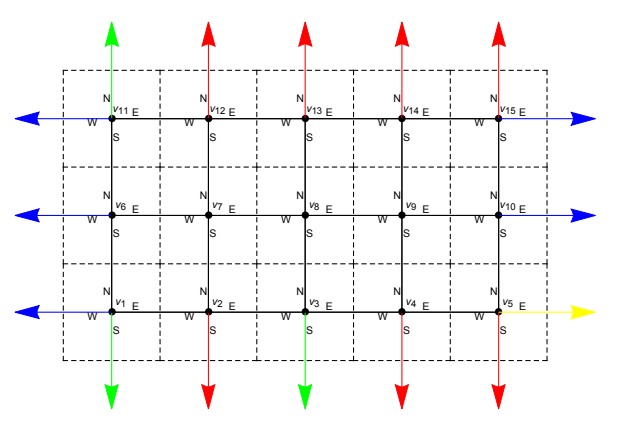

Figure 2: Boundary constraints

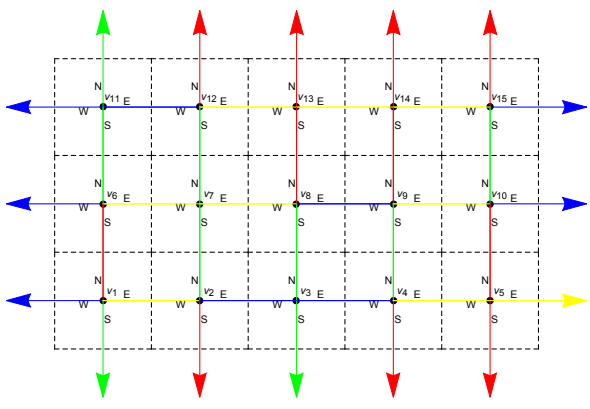

Figure 3: A full extension

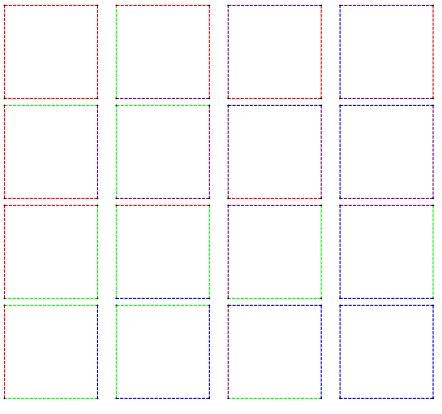

Figure 4: Wang prototiles in $W_{E}$

We are particularly interested in finding a full extension $\tau: E \rightarrow C$ of a given tiling $\beta$ : $N\left(v_{0}\right) \rightarrow C$, which we regard as boundary constraints. Edges in $N\left(v_{0}\right)$ are called constrained legs and those in $E \backslash N\left(v_{0}\right)$ are called free legs. Given boundary constraints $\beta: N\left(v_{0}\right) \rightarrow C$, the tiling problem with $\beta$ is said to be solved if we find a full extension of $\beta$. A tiling problem is said to be always solvable when there exists a full extension $\tau: E \rightarrow C$ of any tiling $\beta: N\left(v_{0}\right) \rightarrow C$. Given a tiling $\tau: F \rightarrow C$, we informally say to tile $v \in V$ with $w \in W$ when we can extend $\tau$ by assigning $\tau\left(e_{i}(v)\right)=w_{i}(1 \leq i \leq \operatorname{deg}(v))$.

Remark 3. This formulation encompasses tiling of non-planar graphs such as a surface in the three dimensional space ([7]). For example, a periodic tiling of a planar grid can be regarded as a tiling of a grid graph on the torus.

Example 4. For $n$ and $m$ be natural numbers, we consider a rectangular region

$$
\begin{aligned}
& V=\left\{v_{i, j} \mid 1 \leq j \leq n, 1 \leq i \leq m\right\}, \text { and } \\
& E=\left\{\left(v_{i, j}, v_{i+1, j}\right) \mid 0 \leq i \leq m, 1 \leq j \leq n\right\} \cup\left\{\left(v_{i, j}, v_{i, j+1}\right) \mid 1 \leq i \leq m, 0 \leq j \leq n\right\},
\end{aligned}
$$

where we set $v_{i, j}=v_{0}$ for $i=0, i=m+1, j=0$, or $j=n+1$. For each $v_{i, j} \in V$, the set of edges $N\left(v_{i, j}\right)$ adjacent to $v_{i, j}$ consists of four edges $e_{E}\left(v_{i, j}\right)=\left(v_{i, j}, v_{i+1, j}\right), e_{N}\left(v_{i, j}\right)=\left(v_{i, j}, v_{i, j+1}\right), e_{W}\left(v_{i, j}\right)=\left(v_{i, j}, v_{i-1, j}\right)$, and $e_{S}\left(v_{i, j}\right)=\left(v_{i, j}, v_{i, j-1}\right)$. The order of $N\left(v_{i, j}\right)$ is given by $e_{E}, e_{N}, e_{W}$, and $e_{S}$. These data define a board $G_{m, n}=\left(V \cup\left\{v_{0}\right\}, E\right)$.

Let $C=\{$ Red, Blue, Purple, Green $\}, W_{E}$ be as in Figure 4 , and consider the tiling problem $\left(G_{5,3}, C, W_{E}\right)$. We set boundary constraints $\beta: N\left(v_{0}\right) \rightarrow C$ as in Figure 2. We note that edges connected to $v_{0}$ are denoted by arrows in the Figure. Also we consider double edges between $v_{0}$ and $v_{1,1}, v_{5,1}, v_{1,3}$ and $v_{5,3}$ which may have different colours. An example of full extension of $\beta$ is given by $\tau: E \rightarrow C$ defined as in Figure 3 .

Example 5. Here we give an example of a board which is not a simple graph. For natural numbers $n$ and $m$, we consider again a rectangular region

$$
V=\left\{v_{i, j} \mid 1 \leq j \leq n, 1 \leq i \leq m\right\} .
$$

This time however the edge set is given as follows: for each $v_{i, j} \in V$, the set of adjacent edges is given by

$$
N\left(v_{i, j}\right)=\left\{e_{E N E}, e_{N E}, e_{N N E}, e_{N N W}, e_{S E}, e_{W N W}, e_{W S W}, e_{S W}, e_{S S W}, e_{S S E}, e_{N W}, e_{E S E}\right\},
$$

where the target of each edge is respectively

$$
v_{i+1, j}, v_{i+1, j+1}, v_{i, j+1}, v_{i, j+1}, v_{i-1, j+1}, v_{i-1, j}, v_{i-1, j}, v_{i-1, j-1}, v_{i, j-1}, v_{i, j-1}, v_{i+1, j-1}, v_{i+1, j} .
$$


(See Figure 5.) Here our convention is $v_{i, j}=v_{0}$ for $i=0, i=m+1, j=0$ or $j=n+1$. These data define a board $G_{m, n}^{\prime}=\left(V \cup\left\{v_{0}\right\}, E=\bigcup_{v \in V} N(v)\right)$. The corner Wang tiling introduced in [11] is a special instance of tiling problem $\left(G_{m, n}^{\prime}, C, W_{C}\right)$, where $W_{C}=\left\{\left(c_{i}\right) \in C^{12} \mid c_{3 k+1}=c_{3 k+2}=\right.$ $\left.c_{3 k+3}, k=0,1,2,3\right\}$. In Figure 5. We show an example of a correspondence between our graphical formalisation and the corner Wang tile in [11].

For example, let $C=\{$ Red, Blue $\}$, and consider a tiling problem $\left(G_{2,3}^{\prime}, C, W_{C}\right)$. Figure 6 gives a solution to the problem.
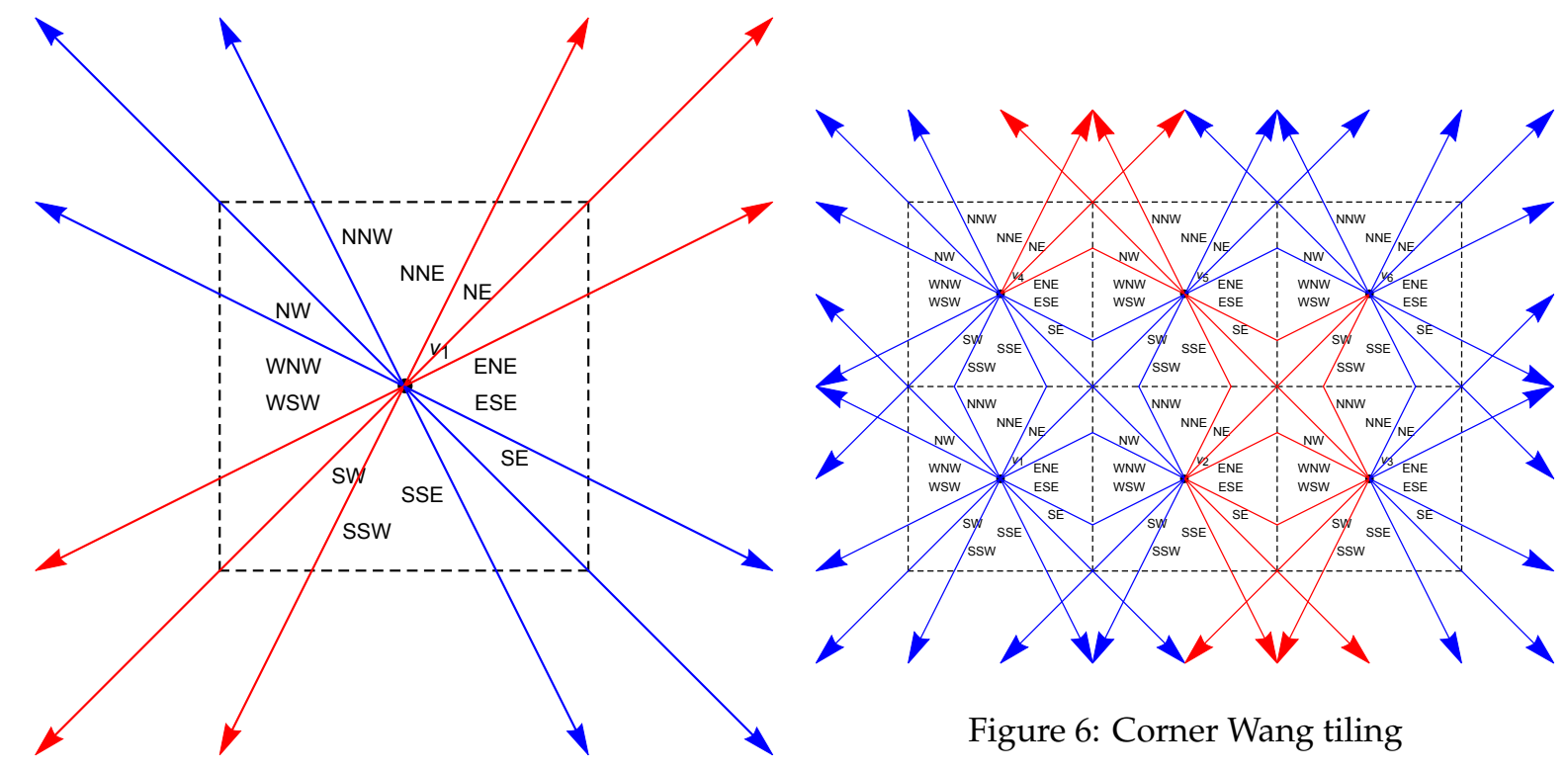

Figure 6: Corner Wang tiling

Figure 5: Corner Wang tile

As mentioned in [11], a corner Wang tiling is equivalent to the ordinary Wang tiling with more colours. Let $\tilde{C}=\{$ Red, Blue, Purple, Green $\}$. We think of a purple edge having blue and red ends, and a green edge having red and blue ends. The tiling problem $\left(G_{2,3}, \tilde{C}, W_{E}\right)$ defined in Example 4 is equivalent to $\left(G_{2,3}^{\prime}, C, W_{C}\right)$. A solution of $\left(G_{2,3}, \tilde{C}, W_{E}\right)$ in Figure 6 corresponds to a solution of $\left(G_{2,3}^{\prime}, C, W_{C}\right)$ in Figure 7. In Figure 8, we display the solution using usual square Wang tiles and corner Wang tiles.

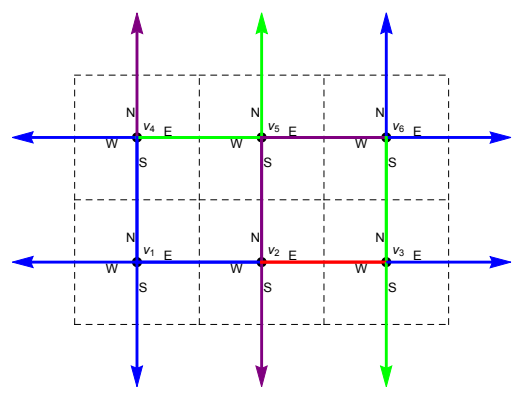

Figure 7: Equivalent Wang tiling

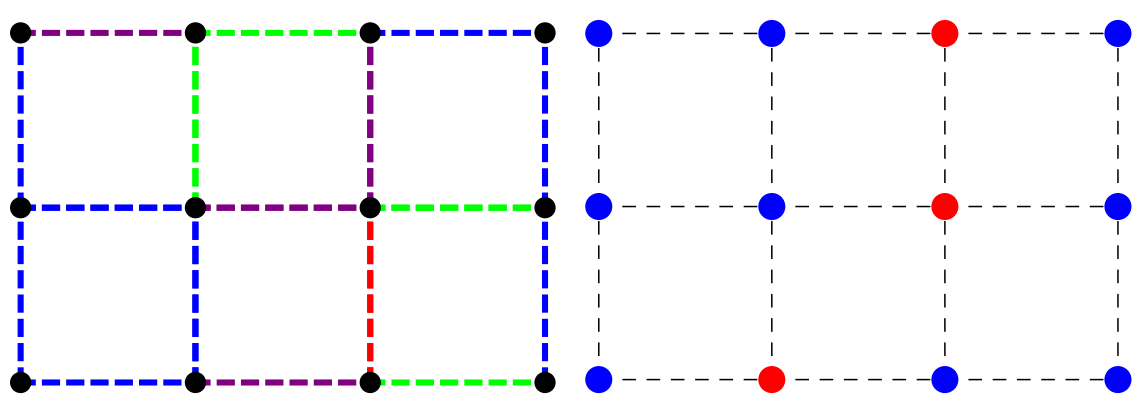

Figure 8: Edge Wang tiling and Corner Wang tiling

Definition 6. For a board $G=\left(V \cup\left\{v_{0}\right\}, E\right)$ and its sub-graph $(U, F)$, where $U \subset V$, the restriction $\left.G\right|_{(U, F)}$ of $G$ is a board obtained by contracting the complement of $(U, F)$ to the constrainer $v_{0}$ : The 
vertex set is the union $U \cup\left\{v_{0}\right\}$ and the edge set is

$$
\left.E\right|_{(U, F)}=F \cup\left\{\left(u, v_{0}\right) \mid u \in U,(u, v) \in E \backslash F\right\} .
$$

That is, an edge not belonging to $F$ is cut into two pieces and each of them is reconnected to $v_{0}$. For a full sub-graph of $G$ on $U \subset V$, we abbreviate the corresponding restriction as $\left.G\right|_{U}$.

In many applications of Wang tiling in computer graphics, the set of Wang prototiles often comes equipped with a nice property, which is rather permissive in terms of tilability.

Definition 7. For a natural number $k$, a set $W$ of prototiles is said to be $k$-sequentially permissive if the following holds:

$$
\pi_{i}^{-}: W_{k} \rightarrow C^{k-1} \text { is surjective for all } 1 \leq i \leq k,
$$

where $\pi_{\bar{i}}$ is the projection dropping the $i$-th factor. In other words, a cell with $k$-legs can always be tiled when it has at least one free leg.

Example 8. Figure 9] shows an example, taken from [3], of a set of prototiles in computer graphics which is 4-sequentially permissive. In practical applications, the colours are replaced by parts of an image to generate textures. Essentially, each side of an edge is one half of an image cut on the diagonal. That way, when tiles are placed adjacently, the transition from one tile to another in the picture is smooth. When all colours are taken from pieces of a given image, and the Wang tiles stitch together the colours, it produces arbitrary large textures from a small example. In [3], this technique is applied to create large Poisson distribution of points by replacing the image parts with carefully designed point distributions.
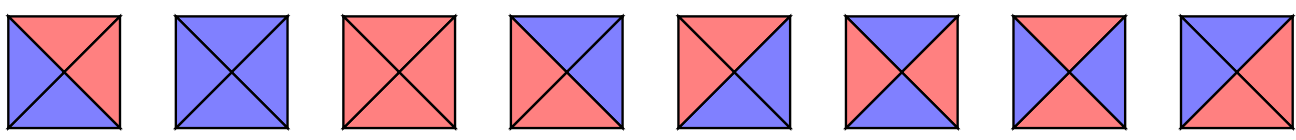

Figure 9: Example of a set of Wang tiles used in computer graphics [3].

The following lemma provides a general reduction strategy to solve tiling problems:

Lemma 9. Let $G$ be a board with the set of cells $V$. Let $W$ be a set of prototiles which is $k$ sequentially permissive (1) for all $k \in\{\operatorname{deg}(v) \mid v \in V\}$. A tiling problem $(G, C, W)$ is always solvable when there exists a restriction $\left.G\right|_{U}$ such that $\left(\left.G\right|_{U}, C, W\right)$ is always solvable.

Proof. Pick a cell $v \in V$ which is adjacent to $U$, and consider $\left(\left.G\right|_{U \cup\{v\}}, C, W\right)$. The vertex $v$ has at least one free leg, which is connected to $U$. So we can tile $v$ by (1) for any $\beta: N\left(v_{0}\right) \rightarrow C$. Now we are left with $\left(\left.G\right|_{U}, C, W\right)$ with extended constraints $\bar{\beta}$, which can be fully extended by assumption. Since $V$ is finite, the assertion follows by induction.

In practice, we proceed in the "opposite" way; we first construct a spanning tree of $\left.G\right|_{V \backslash U}$ and tile it from leaves by (1), and then finally cells in $U$ are tiled by assumption (see \$4). 


\section{Brick Wang tiles}

From now on, we focus on a special set of prototiles which we call the brick Wang tiles. We give a necessary and sufficient condition for a tiling problem to be always solvable.

The brick Wang tiles were introduced in [6] to model wall patterns. Each tile represents how corners of four bricks meet. It is assumed that the edges of the bricks are axis aligned and that each tile is traversed with a straight line, either vertically or horizontally. For aesthetic reasons, crosses are forbidden, where all four bricks are aligned and the corresponding tile is traversed by two straight lines. In this model, the colour in the Wang tiles indicates the position of the edge of the brick on the side of the tile. The formal definition is given as follows:

Definition 10. We always assume $\# C \geq 3$. The set of brick Wang prototiles $W_{B}$ is defined by

$$
W_{B}=\left\{\left(c_{1}, c_{2}, c_{3}, c_{4}\right) \in C^{4} \mid\left(c_{1}=c_{3} \wedge c_{2} \neq c_{4}\right) \text { or }\left(c_{1} \neq c_{3} \wedge c_{2}=c_{4}\right)\right\}
$$

It is easy to see that $W_{B}$ is 4-sequentially permissive (1).

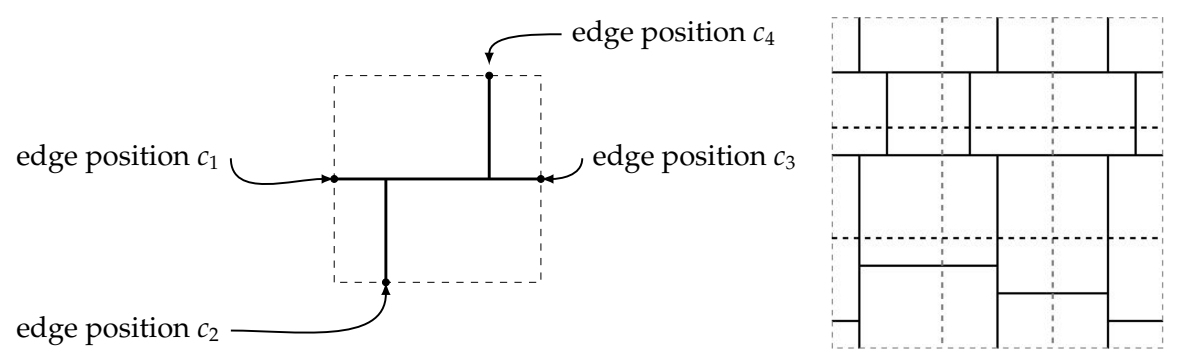

Figure 10: A brick Wang tile and a tiling of $3 \times 3$-board

In the previous work [6, 13], only rectangular regions are considered. Here, we deal with any planar region possibly with holes. In practice, this allows the user to manually tile a part of the region, and leave the rest to be automatically completed by our algorithm in $\$ 4$. See Example 16.

Definition 11. Consider the graph $\bar{G}=\left(V \cup\left\{v_{0}\right\}, E\right)$, where

- $V=\{(i, j) \in \mathbb{Z} \times \mathbb{Z}\}$

- $E=\left\{\left((i, j),\left(i^{\prime}, j^{\prime}\right)\right) \in V \times V|| i-i^{\prime}|+| j-j^{\prime} \mid=1\right\}$.

The set $N((i, j))$ of edges adjacent to a vertex $(i, j)$ are given ordering by

$$
(((i, j),(i+1, j)),((i, j),(i, j+1)),((i, j),(i-1, j)),((i, j),(i, j-1)) .
$$

A board is said to be square grid if it is a restriction of $\bar{G}$ to a finite sub-graph.

We give a simple necessary and sufficient condition for a square grid board $G$ to be always solvable with $W_{B}$. A path is an ordered sequence of distinct cells $\left(v_{1}, v_{2}, \ldots, v_{l}\right)$ where consecutive cells are connected by an edge. A cycle is an ordered sequence of cells $\left(v_{1}, v_{2}, \ldots, v_{l-1}, v_{l}=v_{1}\right)$ where all $v_{i}$ are distinct, and consecutive cells are connected by an edge. A cell $v_{i}$ in a path or a cycle is said to be straight if edges $e_{j}, e_{k} \in N\left(v_{i}\right)$ connected to consecutive cells satisfy $|j-k|=2$ with the ordering of $N\left(v_{i}\right)$. Corner cells in a path or a cycle are those that are not straight. 
Lemma 12. A tiling problem $\left(G, C, W_{B}\right)$ for a square grid board $G$ is always solvable when the full sub-graph on the cells is a cycle.

Proof. Let $\beta: N\left(v_{0}\right) \rightarrow C$ be any colouring of the constrained legs, and $\left(v_{1}, \ldots, v_{l}=v_{1}\right)$ be the full sub-graph of $G$ on the cells. For a cell $v_{k}$, we say the edge connected to $v_{k-1}$ the incoming leg, denoted by in $\left(v_{k}\right)$, and the edge connected to $v_{k+1}$ the outgoing leg, denoted by out $\left(v_{k}\right)$.

First, we can assume there is no straight cells whose constrained legs are given two different colours since they have no effect. Denote by $L_{a, b}$ a corner cell whose constrained leg opposite to the incoming leg is coloured with $a \in C$ and the outgoing leg with $b \in C$. Also, denote by $S$ a straight cell whose constrained legs are coloured with the same colour. These serve as a kind of logical gate:

- $\left\{\begin{array}{l}\tau\left(\operatorname{in}\left(C_{a, b}\right)\right)=a \Rightarrow \tau\left(\operatorname{out}\left(C_{a, b}\right)\right) \neq b \\ \tau\left(\operatorname{in}\left(C_{a, b}\right)\right) \neq a \Rightarrow \tau\left(\operatorname{out}\left(C_{a, b}\right)\right)=b\end{array}\right.$

- $\operatorname{in}(S) \neq \operatorname{out}(S)$.

We prove the assertion by case by case analysis. Note that we can change the ordering of $\left(v_{1}, \ldots, v_{l}=v_{1}\right)$ cyclically, or reverse it.

- Assume that $v_{1}=S, v_{2}=C_{a, b}$. Set $\tau\left(\operatorname{out}\left(C_{a, b}\right)\right)=b$ and tile $\left(v_{3}, v_{4}, \ldots, v_{l-1}\right)$ sequentially by (1). If out $\left(v_{l-1}\right)$ is coloured with $c$, choose a colour for in $(S)$ which is different from $c$. By choosing a colour for out $(S)$ which is different from both $c$ and $a$, we obtain a solution.

- From the previous case, we can now assume that there is no straight cell. Assume that $v_{1}=C_{a, b}, v_{2}=C_{c, d}$ with $b \neq c$. Set $\tau\left(\operatorname{out}\left(C_{c, d}\right)\right)=d$ and tile $\left(v_{3}, v_{4}, \ldots, v_{l-1}\right)$ sequentially by (1). If $\tau\left(\operatorname{out}\left(v_{l-1}\right)\right)=a$, choose a colour for out $\left(C_{a, b}\right)$ which are different from both $b$ and $c$. If $\tau\left(\operatorname{out}\left(v_{l-1}\right)\right) \neq a$, set $\tau\left(\operatorname{out}\left(C_{a, b}\right)\right)=b$. This gives a solution.

- Now, the only remaining case is when $\left(v_{1}, v_{2}, \ldots, v_{l-1}\right)=\left(C_{a_{1}, a_{2}}, C_{a_{2}, a_{3}}, \ldots C_{a_{l-1}, a_{1}}\right)$. Note that $l-1$ should be even since the board is a square grid. Set $\tau\left(i n\left(C_{a_{i}, a_{i+1}}\right)\right)=a_{i}$ and $\tau\left(\operatorname{out}\left(C_{a_{i}, a_{i+1}}\right)\right) \neq a_{i+1}$ for odd $i$. This gives a solution.

Combining Lemma 9 with Lemma 12 , we obtain

Theorem 13. A tiling problem $\left(G, C, W_{B}\right)$ for a square grid board $G$ is always solvable if and only if the full sub-graph on the cells contains a cycle.

Proof. We only have to show the "only if" part. Given any board $G$ whose full sub-graph on $V$ is a tree, we construct $\beta: N\left(v_{0}\right) \rightarrow C$ which cannot be fully extended. We proceed by induction on the number of cells \#V. Pick a leaf $v \in V$ and set $G^{\prime}=G_{V \backslash\{v\}}$. Then, by induction, there exists $\beta^{\prime}: N^{\prime}\left(v_{0}\right) \rightarrow C$ which cannot be fully extended in $G^{\prime}$, where $N^{\prime}\left(v_{0}\right)$ is the edges of $G^{\prime}$ adjacent to $v_{0}$. Let $u$ be the cell connected to $v$ with the edge $e$. We can choose the value of $\beta$ for $N(v) \backslash\{e\}$ so that the colour of $e$ is forced to coincide with $\beta^{\prime}(e)$ in order to tile $v$. Then, the resulting $\beta$ cannot be fully extended.

Remark 14 . Note that a $2 \times 2$ region discussed in [6] is a cycle of length four in our language. So the above theorem is a generalisation of the result in [6]. 


\section{The tiling algorithm for brick Wang tiles}

In this section, we will discuss an algorithm to decide what $\beta$ can be extended to a full tiling $\tau: E \rightarrow C$ and how we can obtain one. An implementation is given at [5].

Fix a tiling problem with a finite planar square grid board $G$ with the Brick Wang tiles $W_{B}$ and a number of colours $\# C \geq 3$. Fix also boundary constraints $\beta: N\left(v_{0}\right) \rightarrow C$. Based on Theorem 13, we design an algorithm to decide solubility and to construct a valid tiling of the problem. The outline goes as follows: let $G^{\prime}$ be the full sub-graph on the cells

1. Detect a cycle in $G^{\prime}$ by performing a depth first traversal of $G^{\prime}$. If there is no cycle, use the tree solver given in Lemma 15.

2. Pick a cell $v$ which is adjacent to but not in the cycle. Construct a tiling in a depth-first manner; that is, tile sequentially from the leaves of a spanning tree rooted at $v$ of the connected component of the complement of the cycle.

3. Repeat Step 2 until there is no cell other than those in the cycle.

4. Construct a tiling of the cycle using the procedure of Lemma 12 .

Since the degree of a cell is always four, then the complexity of the cycle extraction is linear in the number of cells in G. Similarly, we will see that all other operations are performed in a linear time with regards to the number of cells in the board.

The remaining task is now to decide and solve a tree-structured board. To this extent, we introduce the condition propagation that will tell how the border colouring put constraints on the inner legs.

A map $f: C \rightarrow\{$ true, false $\}$ of one of the following forms is called a condition:

- (c) It must match a given colour $c ; f(x)=$ true $\Leftrightarrow x=c$.

- $(\neg c)$ It must not match a given colour $c ; f(x)=$ true $\Leftrightarrow x \neq c$.

- $(*)$ It can match any colour; $f(x)=$ true for any $x$.

To each free leg $e$, we assign a condition $f_{e}$ in such a way that a map $\tau: E \rightarrow C$ satisfying $\tau(x)=\beta(x)$ for $x \in N\left(v_{0}\right)$ and $f_{e}(\tau(e))=$ true for all free legs $e$ gives a full extension of $\beta$.

Fix a cell $v$. If for every free leg of $v$ except for one is assigned a condition, a condition for the last free leg is canonically determined by choosing the weakest one (lower in the above list means weaker). We say that the condition is inferred on the last leg. The precise rule of this is described in Figure 11. For a tree rooted at $v$, we can propagate the condition recursively by inferring from the leafs to the root $v$.

Lemma 15. Let $G$ be a board whose full sub-graph on the cells is a tree rooted at $v$. For a tiling problem $\left(G, C, W_{B}\right)$, boundary constraints $\beta: N\left(v_{0}\right) \rightarrow C$ admit a full extension if and only if $v$ can be tiled in such a way that it matches propagated conditions on its legs. Furthermore, any such tiling of $v$ can be extended to a full extension.

Proof. Only if part is trivial by definition.

Observe that the inferred conditions are so that for any choice of a matching colour for a free leg of a cell, there exists at least one colouring of all other free legs which gives a valid tiling of the cell.

Tile $v$ in such a way that it matches propagated conditions on its legs. Consider the connected components obtained by removing $v$ from $G$, with boundary constraints induced by $\beta$ and the tiling of $v$. Now the proof goes by induction on the number of cells. 
The algorithm is illustrated in Figure 12. Since each cell is visited at most four times, it has a linear complexity with respect to the number of cells.
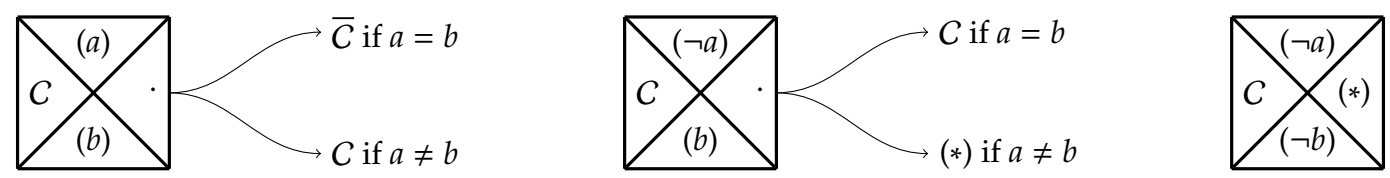

Figure 11: Propagation of conditions for cells in a tree. When some of the legs of the cell are on the boundary, we consider them input legs with a condition $(c)$ where $c$ is the colour of the leg. $C$ denotes a given condition and $\bar{C}$ its negation, with $\overline{(a)}=(\neg a), \overline{(\neg a)}=(*)$ and $\overline{(*)}=(*)$. When any of input conditions is $(*)$, then the output is $(*)$.

Example 16. Figure 13 shows an example of hand-filled regions (the background, the eyes and the mouth of the smiley), completed with an automatically tiled region (the face of the smiley).

\section{Conclusion and Future work}

In this paper, we have proposed a graph theoretical approach to the Wang tiling problem. We have introduced a property of Wang tile sets which is often possessed by those appearing in computer graphics applications. For the class of Wang prototile sets with this property, we have devised a general strategy to reduce a tiling problem to its sub-problem. Then, we used the general result to give a linear algorithm for the tiling of planar regions with the brick Wang tiles. In future work, we would like to consider tiling problems on hexagonal/triangular and irregular polygonal surface meshes, and also volumetric meshes, which would be useful for texturing and point sampling on meshes. Also, we would like to discuss enumeration of all valid tilings for a given problem.

\section{Acknowledgements}

We are grateful to the anonymous referees for their fruitful comments. The second named author was partially supported by JST PRESTO Grant Number JPMJPR16E3, Japan.

\section{References}

[1] The Coq Proof Assistant, https://coq .inria.fr/.

[2] R. Berger. The undecidability of the domino problem. Memoirs of the American Mathematical Society, 66:72, 1966.

[3] M. F. Cohen, J. Shade, S. Hiller, and O. Deussen. Wang Tiles for Image and Texture Generation. ACM Transaction on Graphics, 22(3):287-294, 2003.

[4] K. Culik. An aperiodic set of 13 Wang tiles. Discrete Mathematics, 160(1-3):245-251, 1996.

[5] A. Derouet-Jourdan, Implementation of a linear algorithm for Brick Wang tiling, https: //github.com/KyushuUniversityMathematics/LinearWang

[6] A. Derouet-Jourdan, Y. Mizoguchi, and M. Salvati. Wang Tiles Modeling of Wall Patterns. In Mathematical Progress in Expressive Image Synthesis III, 71-81, Springer Singapore, 2016. 


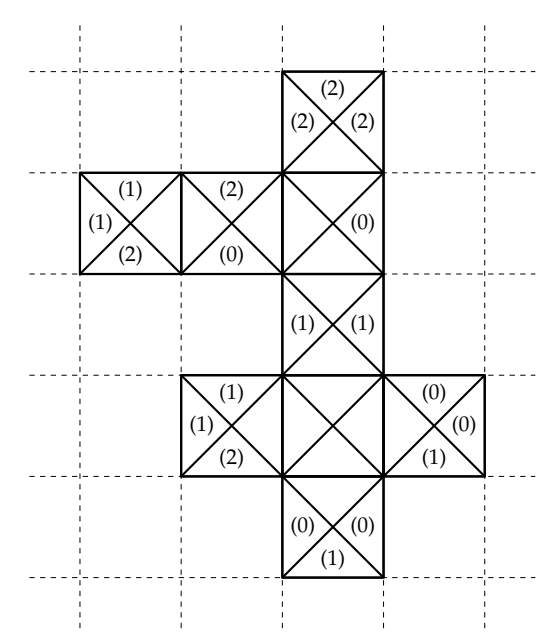

(a)

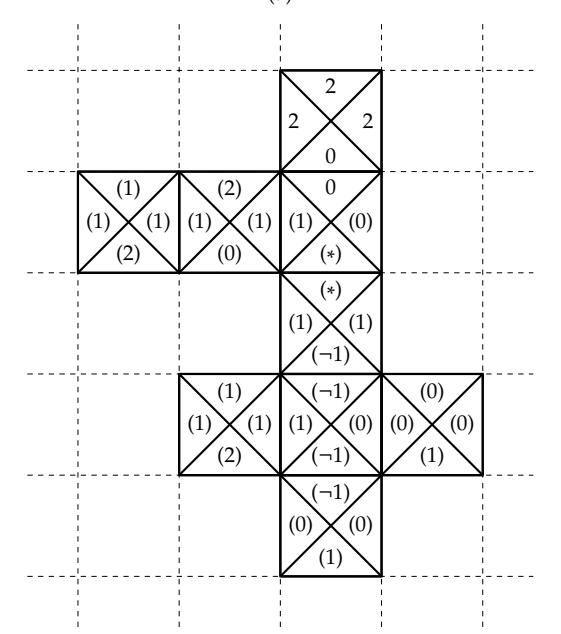

(d)

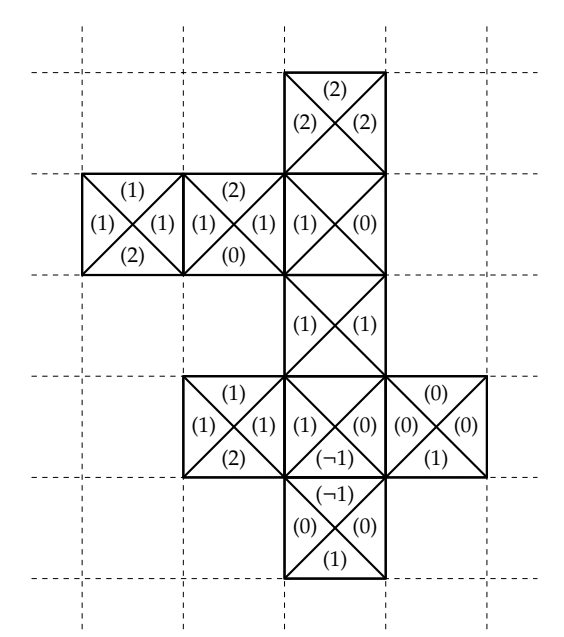

(b)

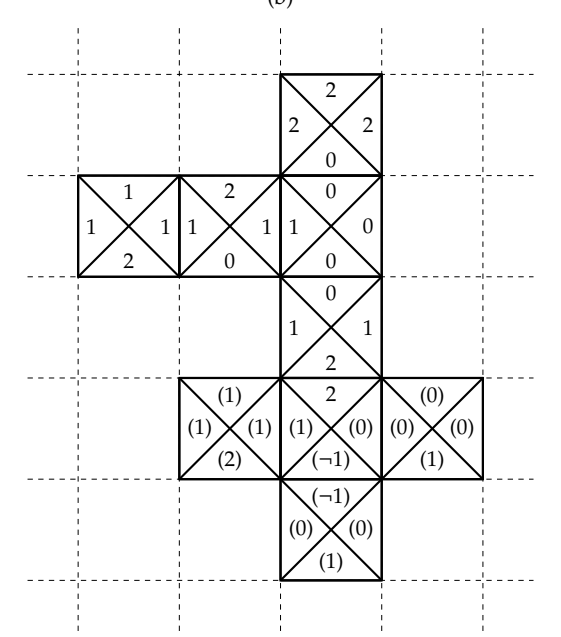

(e)

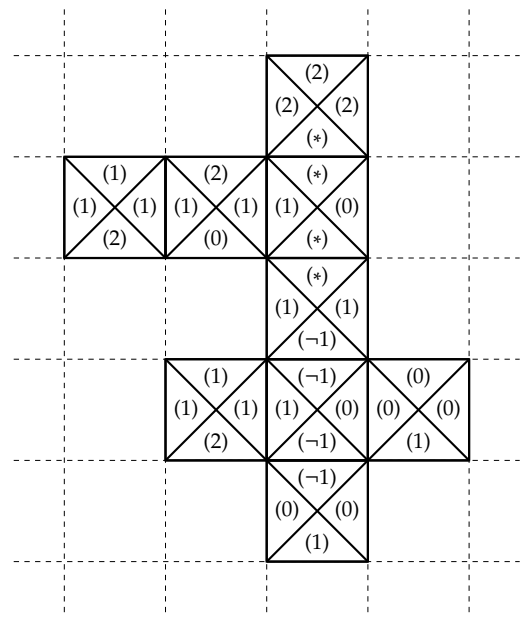

(c)

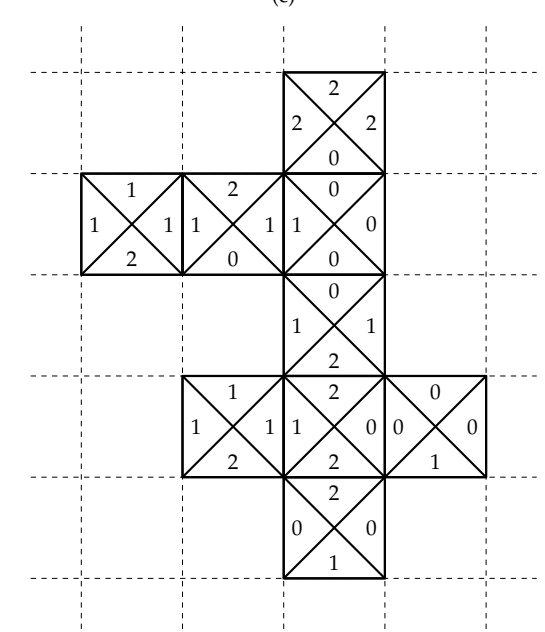

(f)

Figure 12: Illustration of the tree solver. First, (a) we pick input legs on the leaves of the tree and an output leg on the root of the tree (here it is the top cell). Then, we propagate the conditions to the nodes in the tree (b) and to the root (c). If the conditions on the root are compatible, we propagate the solution back from the root (d) to the nodes (e) and the leaves (f).

[7] Chi-Wing Fu and Man-Kang Leung. Texture tiling on arbitrary topological surfaces using wang tiles. In Proceedings of the Sixteenth Eurographics conference on Rendering Techniques (EGSR '05), Eurographics Association, Aire-la-Ville, Switzerland, Switzerland, 99-104, 2005.

[8] E. Jeandel and M. Rao. An aperiodic set of 11 Wang tiles. http://arxiv.org/abs/1506.06492, 2015.

[9] J. Kari. A small aperiodic set of Wang tiles. Discrete Mathematics, 160(1-3):259-264, 1996.

[10] J. Kopf, D. Cohen, O. Deussen, and D. Lischinski. Recursive Wang Tiles for Real-Time Blue Noise. ACM Transaction on Graphics, 25(3):509-518, 2006.

[11] A. Lagae, P. Dutré. A Alternative for Wang Tiles: Colored Edges versus Colored Corners. ACM Tarnsactions on Graphics, 25(4):1442-1459, 2006. 

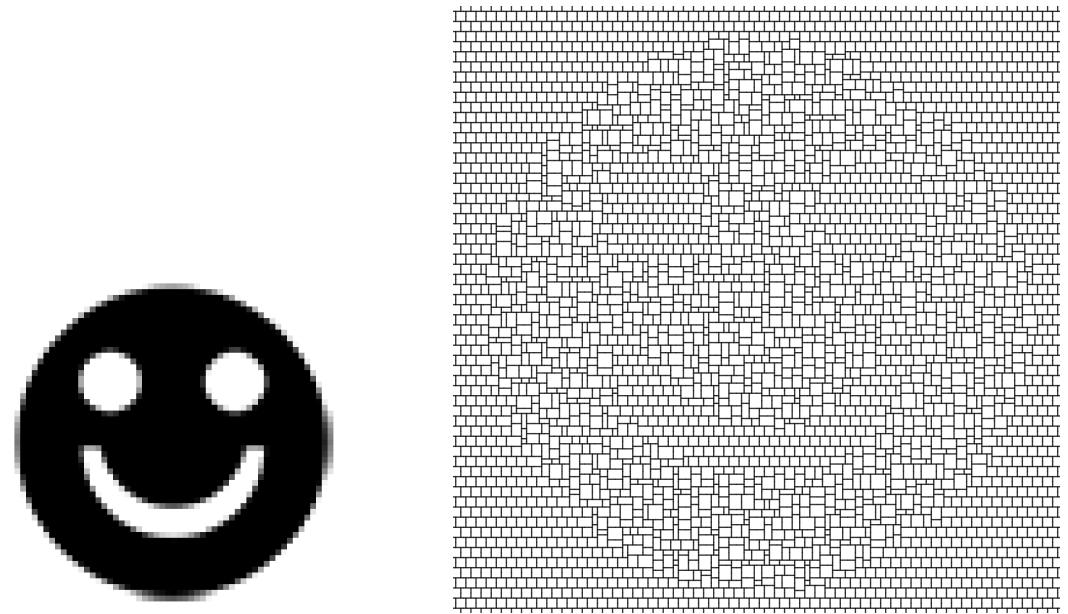

Figure 13: Example of a tiling of a given pattern (top image). The background, the eyes and the mouth are tiled by hand with a regular pattern and the rest (face of the smiley) is tiled automatically.

[12] H. Lewis. Complexity of solvable cases of the decision problem for predicate calculus. Proc. 19th Symposium on Foundations of Computer Science, 35-47, 1978.

[13] T. Matsushima, Y. Mizoguchi, and A. Derouet-Jourdan. Verification of a brick Wang tiling algorithm. in the proceedings of SCSS2016, 2016.

[14] J. Stam. Aperiodic texture mapping. Technical Report R046, European Research Consortium for Informatics and Mathematics (ERCIM), 1997.

[15] H. Wang. Proving theorems by pattern recognition-II. Bell System Technical Journal, 40(1):1$41,1961$. 